\title{
Effect of an Arm Swing on Countermovement Vertical Jump Performance in Elite Volleyball Players
}

\section{FINAL}

\author{
by \\ Frantisek Vaverka ${ }^{1}$, Daniel Jandačka ${ }^{1}$, David Zahradník ${ }^{1}$, Jaroslav Uchytil ${ }^{1}$, \\ Roman Farana ${ }^{1}$, Matej Supej², Janez Vodičar ${ }^{2}$
}

The aim of this study was to determine how elite volleyball players employed the arm swing (AS) to enhance their jump performance. The study assessed how the AS influenced the duration and magnitude of the vertical ground reaction force (VGRF) during the main phases (preparatory, braking and accelerating) of the countermovement vertical jump (CMVJ), the starting position of the body at the beginning of the accelerating phase and the moment when the AS began contributing to increasing the jump height. Eighteen elite volleyball players performed three CMVJs with and without an AS. Kinetics and kinematics data were collected using two Kistler force plates and the C-motion system. The time and force variables were evaluated based on the VGRF, and the position of the body and the trajectory of the arm movement were determined using kinematic analysis. The AS improved the CMVJ by increasing the jump height by $38 \%$ relative to jumping without an AS. The AS significantly shortened the braking phase and prolonged the accelerating phase, however, it did not influence the preparatory phase or the overall jump duration. The AS also significantly increased the average force during the accelerating phase as well as the accelerating impulse. The AS upward began at $76 \%$ into the overall jump duration. The AS did not influence the body position at the beginning of the accelerating phase. These findings can be used to improve performance of the CMVJ with the AS and in teaching beginning volleyball players proper jumping technique.

Key words: jump phases, jump height, VGRF, kinematic analysis, kinetic analysis.

\section{Introduction}

Volleyball players perform vertical jumps frequently during practices and games in various defensive and offensive maneuvers (Kenny and Gregory, 2006; Papageorgiou and Spitzley, 2003). These can be performed as countermovement vertical jumps (CMVJs) with or without an approach step (Ziv and Lidor, 2010) and with different arm positions (Neves et al., 2011). Volleyball players typically utilize a full arm swing whereby the arms are initially swung backward and then moved forward with the elbows fully extended during the CMVJ (Neves et al., 2011). In volleyball the CMVJ is a key movement of the jump serve, jump set, block jump and spike jump (Sheppard et al., 2008).

How the CMVJ is performed is influenced by the combined effects of eccentric and concentric muscle contractions in the

1 - Human Motion Diagnostic Center, University of Ostrava, Ostrava, Czech Republic.

2 - Faculty of Sport, University of Ljubljana, Ljubljana, Slovenia. 
involved joints (ankle, knee and hip), called the stretch-shortening cycle, as well as by whether or not the arms are swung in the takeoff phase (Bosco and Komi, 1979; De Villarreal et al., 2009; Lees et al., 2004a). Numerous studies have demonstrated that appropriate arm movements can increase both the takeoff velocity and the height of the jump (Feltner et al., 2004; Harman et al., 1990; Lees et al., 2004a, 2004b). The arm swing (AS) during the CMVJ can reportedly increase the velocity of the takeoff by at least $6 \%$ relative to without an AS (Harman et al., 1990; Lees et al., 2004a; Luhtanen and Komi, 1978; Shetty and Etnyre, 1989) and increase the jump height (JH) by $15 \%$ or more (Feltner et al., 1999; Harman et al., 1990; Lees et al., 2004a, 2004b).

Many studies have investigated how the AS influences the magnitude of the vertical ground reaction force (VGRF) (Dapena, 1988; Feltner et al., 2004; Lees and Barton, 1996). During vertical jumping, the arms, in terms of the vertical movement of their center of gravity (COG), first move downward toward the ground, thereby reducing the pressure on the ground, which is a negative effect, and then move upward in the takeoff direction, which increases the pressure on the ground and so is a positive effect (Lees and Barton, 1996). The positive influences of the AS on the VGRF curve have been demonstrated in all three of the involved joints (ankle, knee and hip) during the final phase of takeoff (Lees et al., 1996, 2004a). A jump is optimally performed with an AS when the arms are moved in the same direction as the jumping movement itself (Hara et al., 2008).

Our literature review has confirmed the positive effect of the AS on CMVJ performance in various population groups. Yet, we did not find studies investigating the effect of the AS on the three phases of the CMVJ (Figure 1) or the moment when the AS starts contributing to the jump height. We also did not find any studies assessing the influence of the AS on the body position at the beginning of the accelerating phase of the takeoff. The main aims of the present study were to determine the effect of the AS on the phases of the CMVJ, the moment when the AS began to positively influence the jump performance and whether the AS influenced the body position in the beginning of the accelerating phase. We hypothesized that the AS in elite volleyball players had a positive effect during the accelerating phase of the takeoff and did not influence the body position at the beginning of this phase. We assumed that the variability of the moment when AS began to contribute to the jump height would be very low.

\section{Material and Methods}

\section{Participants}

Eighteen elite male volleyball players who were members of the champion team in the top league of the Czech Republic in 2013 (mean \pm SD: age $=27.9 \pm 7.1$ years, body height $=1.92 \pm 0.09 \mathrm{~m}$, body mass $=88.50 \pm 9.46 \mathrm{~kg}$, BMI $=23.92 \pm$ $\left.2.26 \mathrm{~kg} / \mathrm{m}^{2}\right)$ participated in this study. The players were center blockers, receiver hitters and universal players with $6.1 \pm 4.2$ years of experience playing in that league. It could be assumed that these athletes would utilize the AS during the CMVJ in a highly effective manner and that they would perform jumps both with and without an AS at a high level. At the time of testing, the subjects reported no injury that would have prevented their participation in physical activity for more than 2 weeks during the previous 6 months (Dai et al., 2010). The Human Motion Diagnostic Centre Ethics Committee at the Faculty of Education, University of Ostrava approved all procedures used in this study. All the participants signed an informed consent form prior to the commencement of the study.

\section{Procedures}

The experiment was conducted in a biomechanics laboratory within 1 day. After obtaining the approval of each subject to participate in the experiment and making anthropometric measurements, reflective markers for kinematic analysis were applied at appropriate positions on the body. The participants performed their usual warm-up routine, which consisted of a $5 \mathrm{~min}$ jog on a treadmill at a velocity of 2.4-3.2 $\mathrm{m} / \mathrm{s}$, followed by $5 \mathrm{~min}$ of static and dynamic stretching of the lower limbs. After the warm up and prior to testing, two variants of the CMVJ, with and without an AS, were demonstrated to the participants. Each jump began in a standing position motionless. The AS was performed in a manner identical to how the volleyball players usually executed the CMVJ, with the arms first moving in an extension direction at the shoulder joint followed by movement in the direction of flexion. The participants performed three CMVJs 
with the arms fixed at the hips (i.e., without an AS) and three jumps with an AS. The participants were instructed to jump naturally and as high as they could, performing all jumps with maximal effort (Walsh et al., 2004). The negative effects of fatigue were prevented by allowing sufficient rest (a minimum of $30 \mathrm{~s}$ ) between consecutive jumps.

\section{Data collection and analysis}

Twenty-six retroreflective markers (19 $\mathrm{mm}$ in diameter) were attached to the lower body, pelvis, trunk and arms of each participant in positions consistent with a modified version of recommendations from the C-motion company (Rockville, MD, USA). Eight cameras were used $(240 \mathrm{~Hz})$ to monitor the positions of the retroreflective markers, with the obtained position data processed using Visual 3D software (Cmotion). All lower-limb segments were modeled as collections of right circular cones, while the pelvis and trunk were modeled as cylinders (Hanavan, 1964). The local coordinate systems were defined during a calibration trial with the subject standing upright. The coordinate data were lowpass filtered using a fourth-order Butterworth filter with a cutoff frequency of 12 $\mathrm{Hz}$. The velocity of the COG was derived from Visual 3D software (C-motion; Figure 1). The data from two force plates (model 9286AA, Kistler, Winterthur, Switzerland, $1200 \mathrm{~Hz}$ ) measuring the VGRFs for the right and left lower limbs, and from cameras monitoring the positions of the retroreflective markers were collected simultaneously. All force-plate data were lowpass filtered using a fourth-order Butterworth filter with a cutoff frequency of $50 \mathrm{~Hz}$. The final VGRF was calculated as a sum of the VGRF values measured for the right and left lower limbs.

The key moments separating particular phases of the jump were defined based on the function of the VGRF versus time. The velocityversus-time function was evaluated kinematically. The three phases (preparatory, braking and accelerating) of the CMVJ are illustrated in Figure 1. This approach to jump analysis is based on the so-called center-of-mass concept (Bosco and Komi, 1979; Brügemann, 1994). The values of the time and force variables during the defined jump phases were determined using the kinetic analysis of the VGRF curve (Visual 3D software, Cmotion). All of the measured variables, including the preparatory, braking and accelerating phases of the jump takeoff (defined as time periods $\mathrm{tP}, \mathrm{tB}$ and $t A$, respectively), the average force during braking (FBA) and accelerating (FBA) phases, the braking (IB) and accelerating (IA) impulses, as well as the body position in the crucial phases of the CMVJ are displayed and defined in Figure 1.

The body position and AS were defined in the sagittal plane. The ankle angle (AA, shankfoot), knee angle (AK, thigh-shank) and hip angle (AH, trunk-thigh) were numerically evaluated when the body COG was at its lowest position (i.e., at the end of $\mathrm{tB}$ and the beginning of $\mathrm{tA}$; Figure 2). The moment at which AS began to move the arm COG vertically upward was determined from the trajectory of the arm COG in the vertical direction relative to the ground (Figure 2).

The velocity of the body COG at the end of the accelerating phase of the jump (time $\mathrm{t} 3$ in Figure 1) was determined using kinematic analysis, and the $\mathrm{JH}$ was computed using the formula $\mathrm{JH}=v^{2} / 2 g$, where $v$ is the velocity of the body COG and $g$ is the acceleration due to gravity $\left(9.81 \mathrm{~m} \cdot \mathrm{s}^{-2}\right)$. The $\mathrm{JH}$ was assessed for three jumps with an AS and three jumps without an AS, and the reliability of the measured $\mathrm{JH}$ values was calculated for both measured jumping variants, yielding intraclass correlation coefficients of $r=$ 0.973 for jumps with an AS and $r=0.989$ for jumps without an AS. The jumps with the greatest JH were used in the subsequent kinematic and kinetic analyses, and the data from these attempts were used in the final statistical analysis.

\section{Statistical analysis}

Statistical analyses were performed using Statistica (version 10, Statsoft, Tulsa, OK, USA). The presence of data normality was verified using the Kolmogorov-Smirnov test. The differences in variables between jumps with and without an AS were analyzed using paired $t$-tests. The reliability was assessed based on the intraclass correlation coefficient. Statistical significance was considered to be present when $p<0.05$.

\section{Results}

The mean JH was $0.143 \mathrm{~m}(37.7 \%)$ greater for the CMVJ with an AS than for the CMVJ without an AS (Table 1).

IA and IB differed significantly between jumps with and without an AS $(p<0.01)$. IB was significantly greater without an AS than with an 
AS, while IA was significantly greater with an AS than without an AS. Average force FBA did not differ significantly between jumps with and without an AS while average force FAA was significantly greater for jumps with an AS than for jumps without an AS (Table 1).

There were some differences in the duration of the takeoff phases of the CMVJ between jumps with and without an AS (Table 2). The total duration of the takeoff phases and the duration of $\mathrm{tP}$ did not differ significantly between jumps with and without an AS. In contrast, the duration of $\mathrm{tB}$ was significantly longer for jumps without an AS than for jumps with an AS, with the opposite trend of longer duration of $t A$ for jumps with an AS than for jumps without an AS.
The time duration of the jump phases was calculated as a percentage of $\mathrm{tT}$ (total time of the takeoff phase; $\mathrm{tT}=100 \%$ ) (Table 2). The percentage duration of tP did not differ significantly between jumps with and without an AS. The percentage duration of $t B$ was considerably greater for jumps without an AS than for jumps with an AS. The opposite trend was found for the percentage duration of $\mathrm{tA}$, with this being greater for jumps with an AS than without an AS (Table 2). The arm COG started moving vertically upward during the final takeoff phase (i.e., tA) at $75.95 \%$ of the overall jump duration (Table 2). The effect of the AS on the temporal and force variables of the three phases of the CMVJ takeoff is depicted in Figure 3.

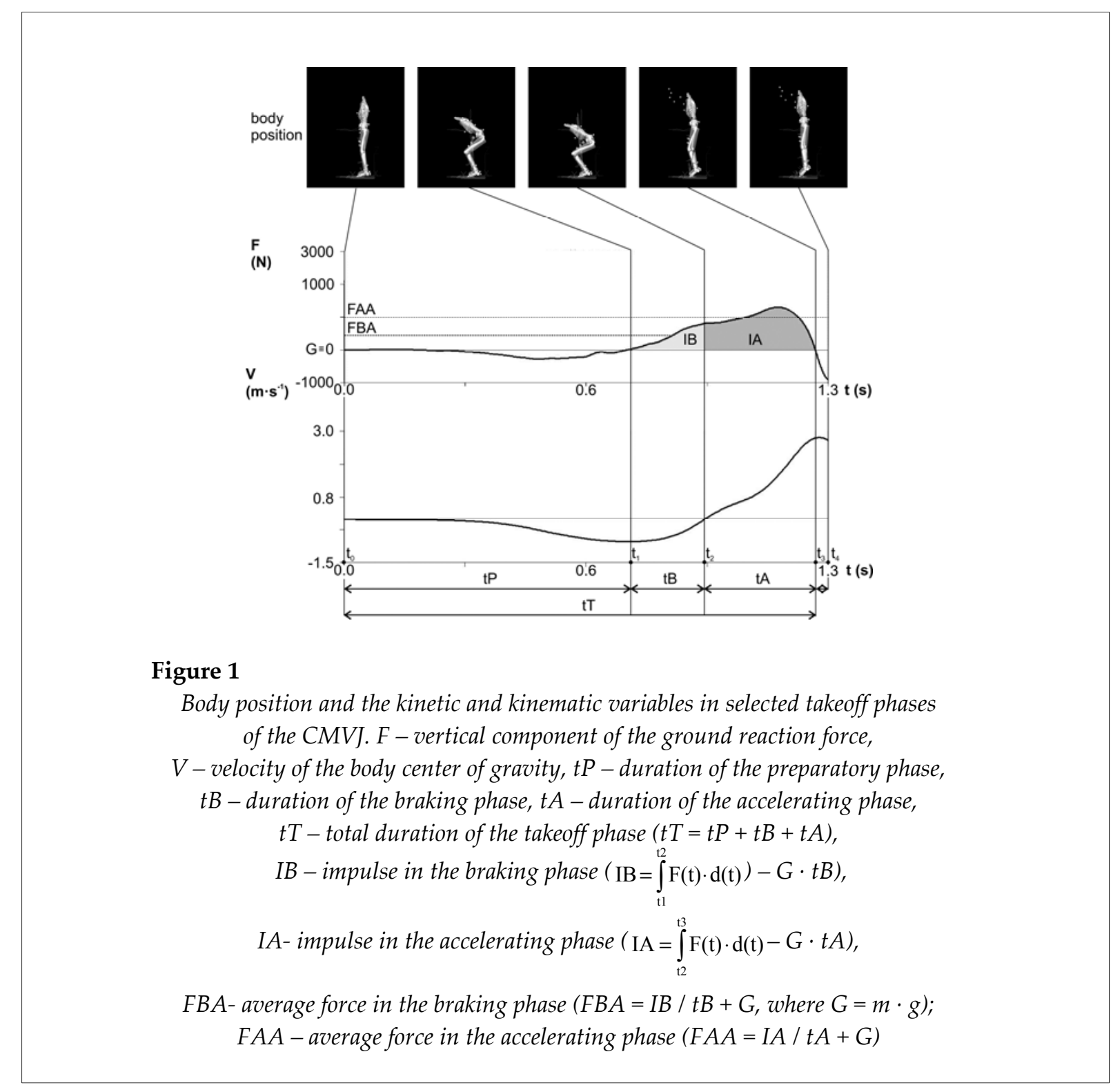




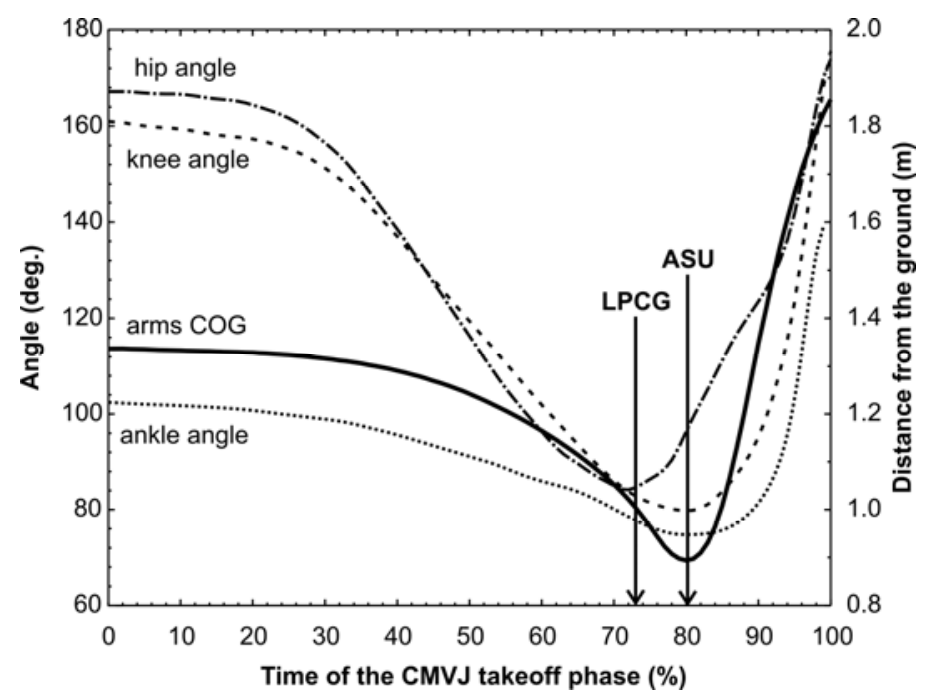

Figure 2

Time courses of the ankle, knee and hip angles and the center of gravity (COG) of the arms in the vertical direction relative to the ground during the CMVJ in a representative individual. Arms COG - the pathway of the centre of gravity of upper extremities in the vertical direction to the ground; LPCG - the lowest position of the centre of gravity of the body; ASU - the beginning of the arm swing upwards.

Table 1

Force variables associated with the two variants of the countermovement vertical jump (CMVJ)

\begin{tabular}{|c|c|c|c|c|c|c|c|}
\hline \multirow{2}{*}{ Variable } & & \multicolumn{2}{|c|}{ No arm swing } & \multicolumn{2}{|c|}{ Arm swing } & \multirow[t]{2}{*}{$t$} & \multirow[t]{2}{*}{$p$} \\
\hline & & Mean \pm SD & $\mathrm{CV} \%$ & Mean \pm SD & $\mathrm{CV} \%$ & & \\
\hline $\mathrm{JH}$ & Jump height (m) & $0.379 \pm 0.057$ & 15 & $0.522 \pm 0.088$ & 17 & 13.99 & $0.001^{* *}$ \\
\hline IB & $\begin{array}{l}\text { Impulse in the braking } \\
\text { phase (Ns) }\end{array}$ & $90.88 \pm 22.13$ & 24 & $79.58 \pm 20.90$ & 26 & 3.80 & $0.001^{* *}$ \\
\hline IA & $\begin{array}{l}\text { Impulse in the } \\
\text { accelerating phase } \\
\text { (Ns) }\end{array}$ & $241.54 \pm 37.67$ & 16 & $283.17 \pm 46.66$ & 16 & 14.05 & $0.001^{* *}$ \\
\hline FBA & $\begin{array}{l}\text { Average force in the } \\
\text { braking phase }(\mathrm{N})\end{array}$ & $1353.35 \pm 247.36$ & 18 & $1408 \pm 217.19$ & 17 & 1.53 & 0.14 \\
\hline FAA & $\begin{array}{l}\text { Average force in the } \\
\text { accelerating phase }(\mathrm{N})\end{array}$ & $1831.85 \pm 344.14$ & 19 & $1882.12 \pm 306.43$ & 16 & 2.05 & $0.05^{*}$ \\
\hline
\end{tabular}

$C V \%$ - coefficient of variation, $t$ - result of a paired $t$-test. ${ }^{*} p<0.05,{ }^{* *} p<0.01$ 


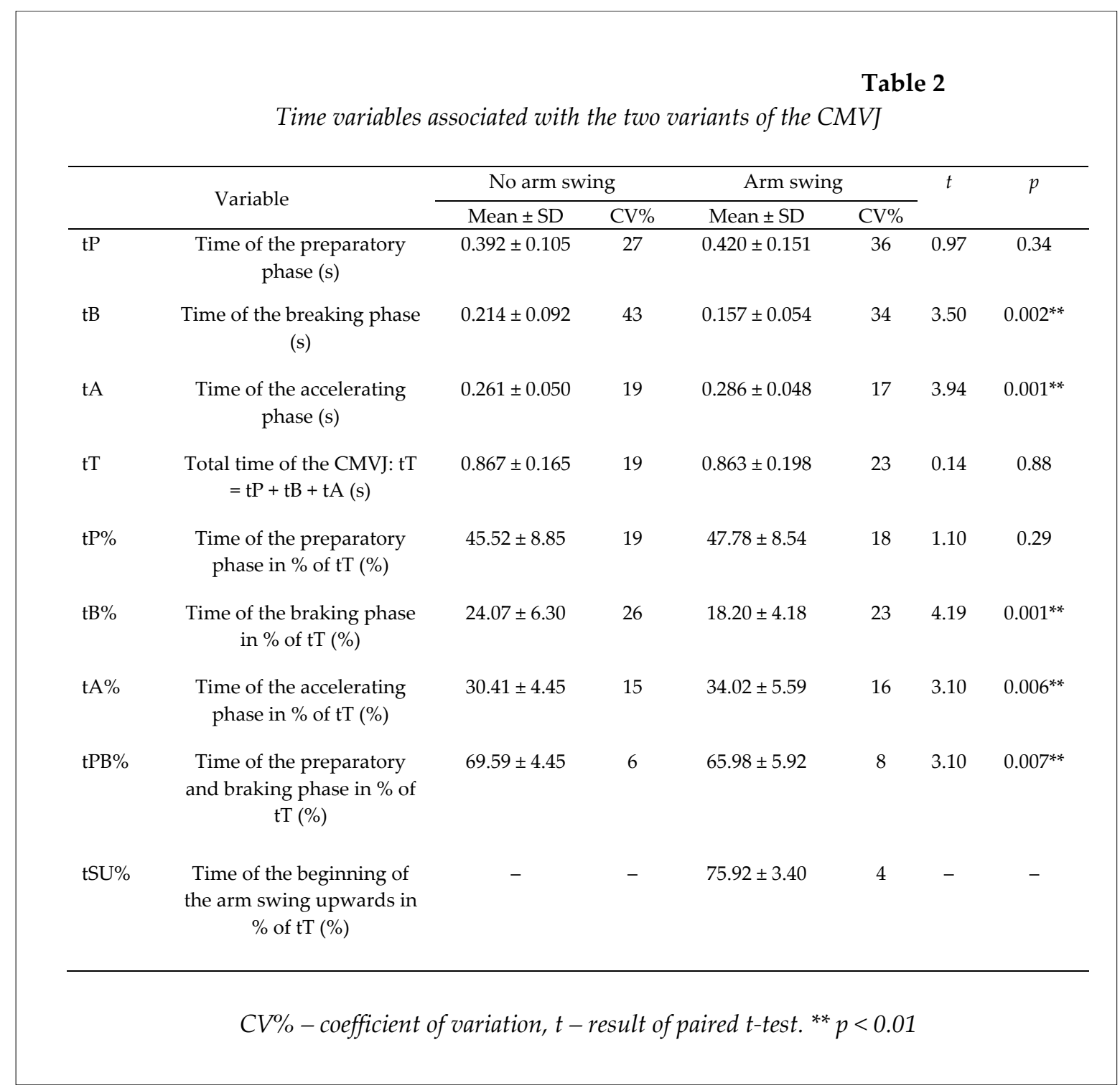

\begin{tabular}{|c|c|c|c|c|c|c|c|}
\hline \multicolumn{8}{|c|}{$\begin{array}{c}\text { Table } 3 \\
\text { Ankle, knee and hip angles at the beginning of the accelerating phase of the CMVJ }\end{array}$} \\
\hline \multirow[t]{2}{*}{ Variable } & \multicolumn{2}{|c|}{ No arm swing } & \multicolumn{2}{|l|}{ Arm swing } & \multicolumn{2}{|l|}{$t$-test } & \multirow[t]{2}{*}{$r$} \\
\hline & Mean \pm SD & $\mathrm{CV} \%$ & Mean \pm SD & $\mathrm{CV} \%$ & $t$ & $p$ & \\
\hline Ankle angle (deg.) & $78.96 \pm 6.43$ & 8 & $78.43 \pm 7.06$ & 9 & 0.84 & 0.41 & $0.93^{* *}$ \\
\hline Knee angle (deg.) & $71.92 \pm 9.74$ & 21 & $72.05 \pm 18.09$ & 25 & 0.07 & 0.94 & $0.90^{* *}$ \\
\hline Hip angle (deg.) & $76.15 \pm 9.74$ & 13 & $78.76 \pm 10.03$ & 13 & 1.57 & 0.13 & $0.75^{* *}$ \\
\hline \multicolumn{8}{|c|}{$\begin{array}{c}\mathrm{CV} \% \text { - coefficient of variation, } t \text { - result of a paired } t \text {-test, } \\
r=\text { Pearson correlation coefficient. }{ }^{* *} p<0.01\end{array}$} \\
\hline
\end{tabular}




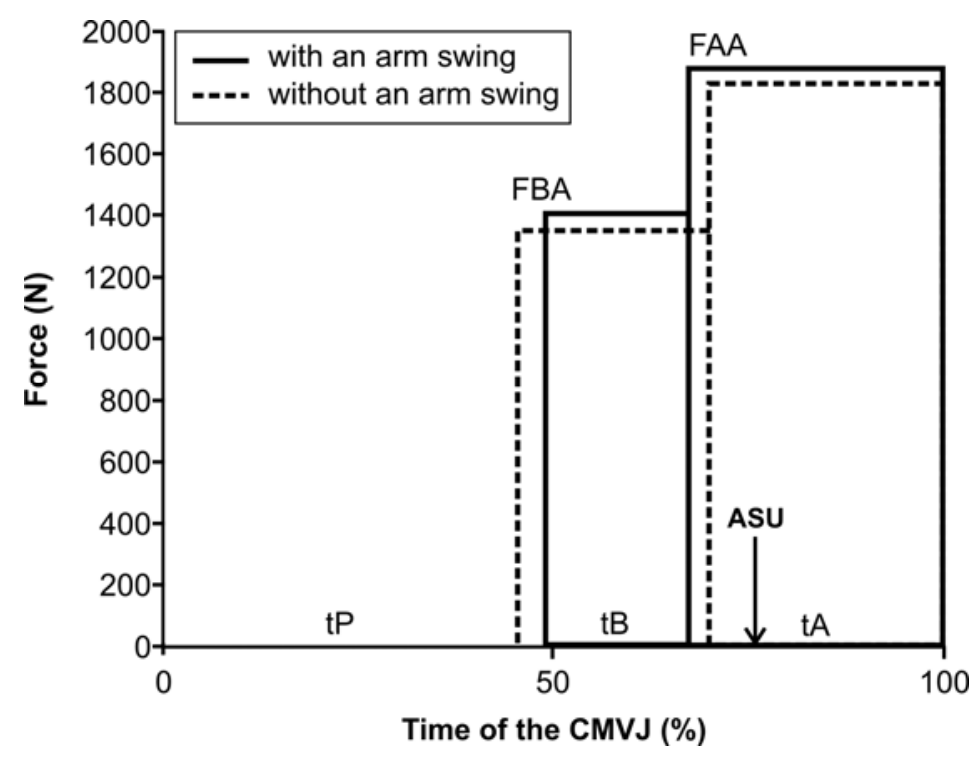

Figure 3

Graphical representation of normalized duration of the preparatory, braking and accelerating phases as well as FBA, FAA and the AS upwards for the CMVJ with and without

an $A S$. $t P$-duration of the preparatory phase, $t B$-duration of the braking phase, $t A$-duration of the accelerating phase, FBA - the average force in the braking phase;

$F A A$ - the average force in the accelerating phase;

ASU - the beginning of the arm swing upwards.

The homogeneity of the measured variables, as quantified by the coefficient of variation $(\mathrm{CV})$, fluctuated between $15 \%$ and $27 \%$ for most of the force variables (Table 1). The variability was larger for IB $(\mathrm{CV}=24-27 \%)$ than for IA $(C V=14-16 \%)$ during both types of the jump. The variability of FBA and FAA was very similar (CV = 16-19\%). The lowest value of the CV in FAA was almost identical for both jump variants $(\mathrm{CV}=11 \%$ and $10 \%$ for jumps with and without an AS, respectively). The CV values for the time variables fluctuated approximately within the same range as for the force variables, with the values only being larger during $\mathrm{tP}$ and $\mathrm{tB}$ $(\mathrm{CV}=27-43 \%)$ (Table 2$)$. We found that the variability was smallest when the AS began in an upward direction $(\mathrm{CV}=4 \%)$.

The angles characterizing the body position at the beginning of the accelerating phase (AA, AK and $\mathrm{AH}$ ) did not differ significantly between jumps with and without an AS (Table 3). The strong correlations between the AA and AK values for jumps with and without an AS ( $r=0.93$ and $r=0.90$ ) indicate that the positions of the lower limbs were essentially identical in the two jump variants. We found a weaker correlation $(\mathrm{r}=$ 0.75 ) between the two variants of the jump for AH.

\section{Discussion}

Jump height was 38\% higher with an AS than without, which indicated that using the arms was highly effective at enhancing the jumping performance in elite volleyball players. Several previous studies (Feltner et al., 1999; Harman et al., 1990; Lees et al., 2004a; Shetty and Etnyre, 
1989) found that the AS increased the CMVJ height from between 15 and $24 \%$, whereas in the present study the increase was $38 \%$. This difference could have been due to the inclusion of top volleyball players in this study adopting a natural AS technique for the CMVJ in volleyball and particularly given the large difference between the observed groups that comprised children (Gerodimos et al., 2008; Harrison and Moroney, 2007), physically active adults and athletes (Harman et al., 1990; Lees et al., 2004a). The evident importance of the AS to CMVJ outcomes was also confirmed by the analysis of the body position at the beginning of the accelerating phase. The hypothesis of the AS not influencing the body position at the beginning of the accelerating phase of the jump was also confirmed (Table 3). The position of the lower limbs was almost identical in both variants of the CMVJ, as also corroborated by the strong correlation between them $(r=0.90-0.93$, and 0.75, Table 3).

There are several possible explanations for a strong contribution of the AS to the JH in elite volleyball players: (a) a great deal of experience in jumping vertically, (b) specific training in proper jumping techniques, and (c) using a highly stable AS method when executing a CMVJ. It follows that the execution of the AS by volleyball players presents a very effective model of arm utilization in the takeoff phase.

The main goal of this study was to determine how the AS influenced the timing and magnitude of the VGRF during the CMVJ. In the literature, the CMVJ takeoff phase is usually further divided into two phases: when the body moves downward (a descent phase) and upward (an ascent phase) (Lees et al., 2004a). Similarly, McGinnis (2013) describes two phases: preparatory and propulsive. Dividing the takeoff phase into three subphases is of practical significance: it is based on a jumping individual's subjective perception of the movement related to the type of muscle contraction of dominant muscle groups during the takeoff, i.e., ankle, knee and hip muscles (Vaverka, 2000; Vaverka et al., 2013). The takeoff phase comprises the preparatory phase (when the body COG accelerates due to the gravitational force toward the ground and muscle force is not being utilized), the braking phase (when the velocity of the COG slows down to a stop, corresponding to eccentric muscle contraction) and the accelerating phase (when the COG accelerates in the direction of the takeoff, corresponding to concentric muscle contraction). $\mathrm{JH}$ is determined by the accelerating phase in which muscles benefit from the eccentric muscle contraction in the braking phase that significantly enhances concentric muscle contraction during the accelerating phase (i.e., the stretch-shortening cycle; Bosco and Komi, 1979). This results in a greater impulse that in turn leads to a greater JH. This concept is a simplification of a takeoff phase as the dominant muscle groups for the ankle, knee and hip joints engage at different time points during the takeoff (Lees et al., 2004a). However, these three phases are easily differentiated using the subjective perception of muscle force exertion (when the subject is decelerating and accelerating), and they are therefore well suited for didactic corrections of jump execution.

Our study showed the effect of the AS on the timing and magnitude of the VGRF only in the braking and accelerating phases of the CMVJ. The AS downward to the ground decreases the pressure on the surface, which shortens the braking phase, as opposed to the accelerating phase when the AS upward increases the pressure on the ground and the tA duration. The AS significantly decreases the IB and increases the IA, what crucially affects JH. The differences between the jumps performed with and without an AS were small both for FBA and FAA, and were statistically significant only for FBA (Table 1). The AS has a positive effect on CMVJ performance when the arms move upward (Lees and Barton, 1996; Lees et al., 1996, 2004a) or generally in the jump direction (Hara et al., 2008). The key factor for a positive contribution of the AS to JH was prolonged duration of the $\mathrm{t} A$, which led to longer duration of force acting in the direction of the jump and therefore, to a higher value of IA. We found that in elite volleyball players, the AS upward began at about $76 \%$ into the total duration of the jump, shortly after the accelerating phase began (which is at the $66 \%$ time point; Figure 2, Table 2). The information about beginning the AS upward at the beginning of the accelerating phase of the jump can be used as a key instruction for ensuring the correct timing of arm movements during the CMVJ. 
The present study has several limitations. First, the players can achieve a higher height of the jump during a volleyball match due to the three steps approach before the CMVJ. Second, the absence of the three steps approach can influence the breaking and acceleration phases of the CMVJ measured in the laboratory. Third, the players executed the CMVJ with and without an AS with their feet in a parallel position. However, the feet are usually not in a parallel position during the take-off in a match. Therefore, future studies should focus on the influence of the feet position on CMVJ performance with an AS, the influence of the steps approach before the CMVJ and also on the influence of augmented feedback on optimal execution of the AS during the CMVJ in young volleyball players.

\section{Conclusion}

A positive influence of the AS on the performance of the CMVJ has been unambiguously demonstrated by an increase in $\mathrm{JH}$ of $38 \%$ relative to jumping without an AS. The AS does not affect the duration of the preparatory phase or the total jump duration, but it significantly shortens the braking phase and prolongs the accelerating phase. The main effect of the AS on jump performance lies in the significant increase in the impulse during the accelerating phase, which depends on the combination of prolonged duration and increased average force during this phase. A vertical AS began at $76 \%$ into the overall takeoff phase duration; that is, soon after starting the accelerating phase (which occurred at $66 \%$ of the total takeoff phase duration). The AS does not influence the starting body position at the beginning of the accelerating phase of the jump. The key factor to ensure that the AS makes an effective contribution to jumping performance is beginning the AS upward shortly after the accelerating phase of the jump begins. The very small variation in the time when the AS upward begins $(\mathrm{CV}=4 \%)$ shows a high and consistent level of utilization of the AS by elite volleyball players. The basic limitation of the study was collecting the CMVJ execution data in a group of elite volleyball players. The appropriate integration of the AS into the takeoff phase in elite volleyball players can be considered to be the optimal model for using the AS to enhance the performance of the CMVJ. The present results further demonstrate that research into the jumping techniques used by elite male volleyball players may help improve their jumping techniques and increase maximal $\mathrm{JH}$, which may be useful for both the coaches and the athletes involved in volleyball.

\section{Acknowledgements}

The study was supported by the grant from The Fund of financial support of sport organizations in Slovenia "Methods of optimization and individualization of the load in selected methods of strength training", No. RR-12-442, 2012.

\section{References}

Bosco C, Komi PV. Potentiation of the mechanical behavior of the human skeletal muscle through prestretching. Acta Physiol Scand, 1979; 106: 467-472

Brügemann GP. Biomechanical considerations on jumping in sports an approach to a fundamental understanding. In Barabás A, Fábián G, (eds.). 12 th International Symposium on Biomechanics in Sports: Proceedings. Budapest: ISBS, 292-294; 1994

Dai B, Sorensen CJ, Gillette JC. The effects of postseason break on stabilometric performance in female volleyball players. Sports Biomech, 2010; 9: 115-122

Dapena J, Chung CS. Vertical and radial motions of the body during the take-off phase of high jumping. Med Sci Sports Exerc, 1988; 20: 290-302

De Villarreal ESS, Kellis E, Kraemer WJ, Izquierdo M. Determining variables of plyometric training for improving vertical jump height performance: A meta-analysis. J Strength Cond Res, 2009; 23: 495-506

Feltner ME, Bishop EJ, Perez CM. Segmental and kinetic contributions in vertical jumps performed with and without an arm swing. Res Q Exerc Sport, 2004; 75: 216-230 
Feltner ME, Fraschetti DJ, Crisp RJ. Upper extremity augmentation of lower extremity kinetics during countermovement vertical jumps. J Sports Sci, 1999; 17: 449-466

Gerodimos V, Zafeiridis A, Perkos S, Dipla K, Manou V, Kellis S. The contribution of stretch-shortening cycle and arm-swing to vertical jumping performance in children, adolescents, and adult basketball players. Pediatr Exerc Sci, 2008; 20: 379-389

Hanavan JP. A mathematical model of the human body. Aerospace Medical Research Laboratory, WrightPatterson Air Force Base; 1964

Hara M, Shibayama A, Arakawa H, Fukashiro S. Effect of arm swing direction on forward and backward jump performance. J Biomech, 2008; 41: 2806-2815

Hara M, Shibayama A, Takeshita D, Hay DC, Fukashiro S. A comparison of the mechanical effect of arm swing and countermovement on the lower extremities in vertical jumping. Hum Mov Sci, 2008; 27: 636648

Harman EA, Rosenstein MT, Frykman PN, Rosenstein RM. The effects of arms and countermovement on vertical jumping. Med Sci Sports Exerc, 1990; 22: 825-833

Harrison AJ, Gaffney S. Motor development and gender effects on stretch-shortening cycle performance. $J$ Sci Med Sport, 2001; 4: 406-415

Harrison AJ, Moroney A. Arm augmentation of vertical jump performance in young girls and adult females. In Menzel HJ, Chagas, MH, (eds.). 25 th International Symposium on Biomechanics in Sports: Proceedings. Ouro Preto, Brasil: ISBS, 130-133; 2007

Kenny B, Gregory C. Volleyball - step to success. Champaign: Human Kinetics; 2006

Lees A, Barton G. The interpretation of relative momentum data to assess the contribution of the free limbs to the generation of vertical velocity in sports activities. J Sports Sci, 1996; 14: 503-511

Lees A, Vanrenterghem J, De Clercq D. Understanding how an arm swing enhances performance in the vertical jump. J Biomech, 2004a; 37: 1929-1940

Lees A, Vanrenterghem J, De Clercq D. The maximal and submaximal vertical jump: Implications for strength and conditioning. J Strength Cond Res, 2004b; 18: 787-791

Luhtanen P, Komi PV. Segmental contribution to forces in vertical jump. Eur J Appl Physiol Occup Physiol, 1978; 38: 181-188

McGinnis PM. Biomechanics of sport and exercise (3rd ed.). Champaign: Human Kinetics; 2013

Neves TJ, Johnson WA, Myrer JW, Seeley MK. Comparison of the traditional, swing, and chicken wing volleyball blocking techniques in NCAA division I female athletes. J Sports Sci Med, 2011; 10: 452-457

Papageorgiou A, Spitzley W. Handbook for competitive volleyball. Oxford: Meyer and Meyer Sport; 2003

Shetty AB, Etnyre BR. Contribution of arm movement to the force components of a maximum vertical jump. J Orthop Sports Phys Ther, 1989; 11: 198-201

Sheppard JM, Cronin JB, Gabbett TJ, McGuigan MR, Etxebarria N, Newton RU. Relative importance of strength, power, and anthropometric measures to jump performance of elite volleyball players. $J$ Strength Cond Res, 2008; 22: 758-765

Vaverka F. Vertical jump - a suitable model for problems in biomechanics and motorics. In Vaverka F, Janura M, (eds.). Proceedings of the conference BIOMECHANICS OF MAN 2000. Olomouc: Palacky University, 213-216; 2000

Vaverka F, Jakubsova Z, Jandacka D, Zahradnik D, Farana R, Uchytil J, Supej M, Vodicar J. The influence of an additional load on time and force changes in the ground reaction force during the countermovement vertical jump. J Hum Kinet, 2013; 38: 191-200

Walsh M, Arampatzis A, Schade F, Brüggemann GP. The effect of drop jump starting height and contact time on power, work performed, and moment of force. J Strength Cond Res, 2004; 18: 561-566

Ziv G, Lidor R. Vertical jump in female and male volleyball players: A review of observational and experimental studies. Scand J Med Sci Sports, 2010; 20: 556-567

\section{Corresponding author: Frantisek Vaverka}

Human Motion Diagnostic Center, University of Ostrava, Varenska 40a, 70103 Ostrava, Czech Republic Phone: +420 777609511; Fax: +420 596120705; E-mail: frantisek.vaverka@gmail.com;

frantisek.vaverka@osu.cz 